\title{
Periodontal health conditions in patients with diabetes in a family medicine unit
}

\begin{abstract}
Introduction: Periodontitis is a multifactorial pathology, due to the continuous presence of subgingival biofilm, which causes chronic inflammation and destruction of the periodontal tissues. The progression of periodontitis is influenced by several causes and risk factors such as the microbial composition of the plaque and systemic diseases such as diabetes.

Objective: To determine the periodontal health conditions presented by diabetic patients in a family medicine unit.

Material and methods: An observational, cross-sectional and prospective study was carried out in 175 adult patients with diabetes who attended the educational program DiabetIMSS at the Family Medicine Unit No.40 in Mexicali, Mexico; from June through August 2018. Periodontal condition and oral hygiene were evaluated using the Community Periodontal Index (CPI) and the Simplified Oral Hygiene Index (OHI-S) respectively. Descriptive statistics were used with measures of central tendency, percentages and frequencies and Fisher's test were applied using the SPSS v.21 program.
\end{abstract}

Results: Vast majority of patients (89.72\%) had periodontal disease and $57.14 \%$ presented regular oral hygiene. Commonly, patients brushed their teeth twice per day $(54.6 \%)$. An association between CPI and OHI-S instrument was established (Fisher's test 27.456, $\mathrm{p}<0.001)$.

Conclusion: The majority of patients with type 2 diabetes have periodontal disease and a regular or poor oral hygiene. We recommend taking preventive measures in oral hygiene in order to prevent disease.

Keywords: periodontitis, diabetes, gingivitis, oral health, disease, oral hygiene, bacterial microorganisms, tooth loss, blindness, kidney failure, heart attacks, stroke, lower limb amputation
Volume 3 Issue 3 - 2019

\author{
Naomi Angulo Barrientos,' María Elena Haro \\ Acosta, ${ }^{2}$ Guadalupe Angulo Espinoza ${ }^{3}$ \\ 'Stomatology intern, Family Medicine Unit (FMU) No.40 of the \\ Mexican Institute of Social Security (IMSS), Mexico \\ ${ }^{2}$ DSc Pediatrician and Teacher in School of Medicine, \\ Universidad Autónoma de Baja California, Mexico \\ ${ }^{3}$ Collaborator, Family Doctor, UMF No.40 of the IMSS, Mexicali, \\ Mexico
}

Correspondence: María Elena Haro Acosta, DSc Pediatrician and Teacher in School of Medicine, Universidad Autónoma de Baja California, Dr. Humberto Torres Sangines Street. S/N Centro Cívico, CP 21000, Mexicali, Baja California, Mexico, Email lenaharo@live.com.mx

Received: March 07, 2019 | Published: May 07, 2019
Abbreviations: WHO, world health organization; CPI, community periodontal index; DMF, decay-missing-filled index; OHI-S, simplified oral hygiene index; IMSS, Mexican institute of social security; FMU, family medicine unit

\section{Introduction}

Periodontal disease is a pathological and chronic inflammation of the gums caused by bacterial microorganisms that reside in a biofilm, which causes destruction of dental insertion structures, such as the periodontal ligament and alveolar bone, which can cause tooth loss. ${ }^{1}$ Diabetes mellitus, is one of the systemic diseases that are considered a risk factor to develop periodontal disease. Periodontal involvement, in turn, can also have a negative effect on the diabetes management and control. ${ }^{1-3}$ The World Health Organization (WHO) defines diabetes mellitus as a state of chronic hyperglycemia, which over time damages: blindness, kidney failure, heart attacks, stroke and lower limb amputation. It can be treated with diet, exercise, medication and regular evaluations by the physician. ${ }^{4}$ The global prevalence of diabetes is expected to increase to 592 million people in $2035 .{ }^{5}$ The WHO has used the Community Periodontal Index (CPI) as an instrument to establish the periodontal health status of countries for in order to plan treatment and control programs regarding periodontitis, as it is a quick and simple instrument to apply. ${ }^{6}$ In Mexico, the Epidemiological Surveillance System of Oral Pathologies concluded that gingival bleeding and dental calculus were common in the country according to CPI measurements. This reflects the lack of oral hygiene and other concomitant factors such as diabetes, obesity and pregnancy. ${ }^{7}$ As a precedent, a study conducted in Zacatecas used the oral epidemiological indexes: decay-missing-filled index (DMF) and Simplified Oral Hygiene Index (OHI-S) to know the oral health in a stomatology clinic. ${ }^{8}$ Given that diabetes is a common diagnosis among Mexican population, and oral health plays an important role in its control, the objective is to determine the conditions of periodontal health in patients with diabetes in a family medicine unit.

\section{Methods}

An observational, cross-sectional, prospective and analytic study was performed in 175 patients with diabetes who attended the group DiabetIMSS from July to August 2018 at the Family Medicine Unit (FMU) No.40 of the Mexican Institute of Social Security (IMSS) in Mexicali, Baja California. All patients who attended the unit during the study period were included. The inclusion criteria were: patients with diabetes who attended the DiabetIMSS group of the UMF 40 , aged 18 to 70 years old and who agreed to participate in the study. The exclusion criteria were: patients already diagnosed and treated for periodontal disease in the six months prior to the study. All the individuals' teeth were examined, using a dental mirror and a periodontal probe. The measuring instruments were: Community Periodontal Index $(\mathrm{CPI})^{9,10}$ and the Simplified Oral Hygiene Index (OHI-S). ${ }^{11}$ The CPI allows detection of the signs of periodontal disease and classifies the stage of involvement in each individual. This index takes into consideration whether gums are healthy or not, if there was bleeding or inflammation, the presence and size of periodontal pockets, degree of bone destruction and dental mobility. It assigns 
a score to each criterion. The state of oral hygiene was estimated using the OHI-S, with the help of plaque disclosing tablets. The final OHI-S score is the sum of the mean dentobacterial plaque and dental calculus. The calculation was made by adding the stained sides and the dental calculus between the examined teeth. Each was assigned a numeric value: 0 is excellent, 0.1 to 1.2 is good, 1.3 to 3.0 is regular and 3.1 to 6.0 are bad. Age, gender, degree of education, occupation, type of diabetes, tooth mobility and oral hygiene measures were also interrogated and logged. The information was analyzed using descriptive statistics, frequencies and percentages and Fisher's test with the support of the SPSS version 21 programs. This research was accepted by the Local Research Committee 201 Mexican Institute of Social Security (IMSS) and follows the guidelines of the Declaration of Helsinki were followed and of Mexico's General Health Law. Informed written consent was obtained and patient confidentiality was protected.

\section{Results}

After applying the inclusion and exclusion criteria, a total of 175 patients were included in the study. Then mean age was $53.83 \pm 11.42$ years. Regarding gender, $70.28 \%(\mathrm{n}=123)$ were women and $29.71 \%(\mathrm{n}=53)$ male. The highest degree of education in $35.4 \%$ was elementary school, $34.3 \%$ middle school $16.5 \%$ high school, $9.1 \%$ held an academic degree and $5.1 \%$ were illiterate. Periodontal disease was present in $62.85 \%$ of female patients and $26.85 \%$ of male patients. Using the CPI, $46.85 \%$ had periodontal pockets sized $4-5$ $\mathrm{mm}, 15.42 \%$ had bleeding in response to light stimulation. Dental mobility was found in $20(11.42 \%)$ patients (Table 1). According to oral hygiene measures, $172(98.28 \%)$ patients brush their teeth on a regular basis; $54.6 \%$ is done twice per a day. The OHI-S's scores were classified either as acceptable (good and excellent categories) or deficient (for regular and bad indicators). According to the final OHI-S score, 130 (74.28\%) patients had a deficient oral hygiene. Out of the remaining $45(27.72 \%)$ patients, $40(22.85 \%)$ classified as good and only $5(2.85 \%)$ as excellent. After applying the Fisher's test, an association between CPI and OHI-S scores was established (Fisher's test 27.456, $\mathrm{p}<0.001$ ) (Table 2).

Table I Frequency of periodontal disease with the CPI instrument

\begin{tabular}{lll}
\hline Community periodontal Index & Frequency & Percentage \\
\hline Healthy & 18 & 10.3 \\
Bleeding & 27 & 15.4 \\
Calculus & 24 & 13.7 \\
Periodontal Bags $4-5 \mathrm{~mm}$ & 82 & 46.9 \\
Periodontal Bags $\geq 6 \mathrm{~mm}$ & 24 & 13.7 \\
Total & 175 & 100 \\
\hline
\end{tabular}

Table 2 Association between CPI and OHI-S

\begin{tabular}{llll}
\hline CPI & IHOS & \multicolumn{2}{l}{ Total } \\
& Acceptable & Deficient & \\
\hline Healthy & II & 7 & 18 \\
Bleeding & 11 & 16 & 27 \\
Calculus & 8 & 16 & 24 \\
Periodontal Bags 4-5 mm & 15 & 67 & 82 \\
Periodontal Bags $\geq 6 \mathrm{~mm}$ & 0 & 24 & 24 \\
Total & 45 & 130 & 175 \\
\hline
\end{tabular}

\section{Discussion}

In the present study, the periodontal condition of diabetic patients attending the educational program Diabet IMSS of the Family Medicine Unit No. 40 was determined. Female patients $(62.86 \%$, $\mathrm{n}=110)$, was similar the study by Peralta et al. ${ }^{12}(62.1 \%, \mathrm{n}=194) .{ }^{12} \mathrm{The}$ mean age was 53.83 years, which is similar to the study by Ochoa et al., ${ }^{13}$ (57.3 years); although in his study, $64.7 \%$ of the patients were type 2 diabetics and $35.9 \%$ type 1 diabetes, ${ }^{13}$ meanwhile, all patients in our study were type 2 diabetes. The $89.72 \%$ of our study group had some extent of periodontal disease; greater than observed by González et al., ${ }^{14}$ was the $68.14 \%$, of 135 patients aged 25 59 years; our study was 18-70 years. The patients with the highest percentage of periodontal involvement were those whose highest degree of education was elementary or middle school, similar to what was observed by González et al. ${ }^{14}$ The most frequent of type of periodontitis in our study was moderate periodontitis, similar to the study by Apaza et al., ${ }^{15}$ where 117 diabetic patients where studied and $48.6 \%$ of them had moderate periodontitis. Contrarily, Macas et al. ${ }^{16}$ found that, amongst their 101 patient study, $39.6 \%$ had mild and $37.6 \%$ had moderate periodontitis, which may be due to the fact that they included type 1 and 2 diabetics. In this study, 54.6\% of patients brush their teeth two times a day, which differs from the findings by Valenzuela et al., ${ }^{17}$ where at least $61.36 \%$ of their patients brushed their teeth at least once a day. According to the OHI-S score, $74.28 \%$ of our patients had deficient buccal hygiene, which correlates to the $71.2 \%$ found by Soca and Martínez, ${ }^{18}$ and is significantly higher than the $37.1 \%$ reported by Trujillo et al. ${ }^{19}$ Previous research has already established that poor oral hygiene increases the risk of presenting periodontitis by two to five times..$^{20}$ The study conducted by Castle, ${ }^{21}$ concludes that it is necessary to prevent and timely treat dental plaque, and teach patients the basics to maintain a good oral hygiene. Unfortunately, in the present study, according to CPI results, most of the patients require specialized treatment, that could consist of deep scaling, root planning or complex surgical treatment. It is important to emphasize oral hygiene measures, especially in diabetic patients, in order to avoid health problems like periodontal disease.

\section{Conclusion}

In our hospital, the majority of patients with type 2 diabetes have some degree of periodontal disease and a regular or bad oral hygiene. Additionally, an association between CPI and OHI-S was observed. It is imperative to create strategies so that diabetic patients attend the stomatology service so that they know their oral health status and be treated in the initial phases of periodontal disease if needed.

\section{Acknowledgments}

We thank Dr. Joan Dautt for her help translating this article.

\section{Conflicts of interest}

The author declares there is no conflict of interest.

\section{References}

1. Fajardo Puig ME, Rodríguez Reyes O, Hernández Cunill M. Diabetes mellitus and periodontal disease: current physiopathological aspects of their relationship. MEDISAN. 2016;20(6):845-850.

2. Aguilar Soto FE, Sosa Morales FJ, Bojórquez Anaya Y, et al. Periodontitis a multifactorial disease: Diabetes Mellitus. RICS. 2017;6(11).

3. Anguiano Flores L, Zerón A. Periodontal diseases and their relationship with systemic diseases. Rev Mex Periodontol. 2015;6(2):77-87. 
4. World Health Organization. Diabetes, Descriptive note October 2018. 2018.

5. International Diabetes Federation. World diabetes day. Brussels: IDF; 2015. 2018.

6. World Health Organization. The WHO Global Oral Health Data Bank. Geneva: World Health Organization. 2003.

7. Epidemiological Surveillance System of Oral Pathologies, 10 years monitoring the oral health of Mexicans. December, 2015. Health System. 2018. p. 1-87.

8. Franco Trejo CS, Medrano Cortes E, Falcón Reyes LP, et al. Nivel de autocuidado y enfermedades bucales más frecuentes en pacientes de una clínica universitaria. RICS. 2017.

9. Russell Periodontal Index WHO REVISED Form (IPC). 2018.

10. Periodontal Status Index. 2018.

11. Gómez Ríos NI, Morales García MH. From the CPO-D and IHOS indexes in students of the Universidad Veracruzana, Mexico. Rev Chil Public Health. 2012;16(1):26-31.

12. Peralta-Álvarez D, Espinosa-Cristóbal LF, Carreón-Burciaga RG, et al. Evaluation of periodontal disease and hyperglycemia associated with type 2 diabetes mellitus in patients from the state of Durango, Mexico. Rev ADM. 2016;73(2):72-80.

13. Ochoa SP, Ospina CA, Colorado KJ, et al. Periodontal condition and dental loss in diabetic patients. Biomedical. 2012;32(1):52-59.
14. González Gutiérrez A, Trasancos Delgado M, González Cordero AE. Behavior of periodontal disease in diabetic patients. Pedro Borrás Astorga Polyclinic. Rev Medical Sciences. 2012;16(1):169-180.

15. Apaza Huanca LM, Pacompia Quispe HJ. Enfermedad periodontal asociado a la diabetes mellitus en pacientes atendidos en el Hospital Carlos Monge Medrano de Juliaca, 2017. Peru. Universidad Andina. 2017.

16. Macas Malla LG, Avila Guazha JA. Periodontal clinical diagnosis in patients with type 1 and type 2 diabetes mellitus attended at the DONUM Foundations and the Diabetes House in the city of Cuenca in the period: January to July 2015. Cuencia, Ecuador. Universidad Cuenca. 2015.

17. Valenzuela Narvaez RV, Mejia C, Valenzuela D. Association of periodontal disease and diabetes mellitus type 2, Peru, Alas Peruanas University. Science and Development. 2017;20(2):33-39.

18. Miguel Soca PE, Santiago Martínez Y. Periodontal disease, inflammation and diabetes mellitus. Gac Med Espirit. 2016;18(3):1-4.

19. Trujillo Sainz ZC, Eguino Ortega E, Paz Paula CM. Oral health status in diabetic patients over 20 years of evolution, Cuba. Rev Medical Sciences of Pinar del Río. 2017;21(1):47-53.

20. Lertpimonchai A, Rattanasiri S, Arj-Ong Vallibhakara S, et al. The association between oral hygiene and periodontitis: a systematic review and meta-analysis. Int Dent J. 2017;67(6):332-343.

21. Castle Bolio R. Need for periodontal treatment in patients with type II diabetes mellitus in the community of Chapab, Yucatán. Rev Odontol Latinoam. 2013;5(2):35-39. 